\title{
P021. Investigation on occipital headache associated with vertigo and vomiting discovers a familial clustering of Chiari I malformation and a "puzzle"
}

\author{
Alessandro Panconesi*, Marco Macucci, Maria L Bartolozzi, Stefania Mugnai, Leonello Guidi \\ From Abstracts from the 1st Joint ANIRCEF-SISC Congress \\ Rome, Italy. 29-31 October 2015
}

A 16-year-old male (patient 4) experienced an episode of bilateral parietal headache, preceded by vertigo and associated with vomiting, lasting about two weeks. An MRI scan performed for a subsequent episode of occipital and neck pain with vomiting and vertigo showed an imaging to the limit of Chiari I malformation (CMI): cerebellar tonsils below the foramen magnum, not reaching posterior arch of $\mathrm{C} 1$, but with obliteration of peribulbar posterior liquoral space, cyst septum pellucidum and cavum vergae. Left facial nerve palsy at the age of 12 , frequent alimentary vomiting and abdominal pain, episodes of exerciseinduced asthma although not confirmed through respiratory tests, were found in the patient's history.

An MRI was performed on his father for otoneurological symptoms characterized by a recurrent sensation of disequilibrium when suddenly changing position, on one occasion preceded by a sensation of right ear pressure (diagnosed as sudden hearing loss). Two years ago he experienced an episode of stabbing occipital headache with shoulder pain irradiation, nausea and phonophobia. The MRI revealed cerebellar tonsils slightly below the foramen magnum, to the limit of CMI. MRI was performed also on his mother (patient 1), who suffers from tension-type headache even for long periods of time (months), showing no alterations, and on his 21-year-old brother (patient 5) suffering from episodic tension-type headache, showing cerebellar tonsils slightly below the foramen magnum, to the limit of CMI.

His mother's interview revealed that her sister (patient 3) has five sons, two of them with CMI: 1) a 13-year-old male (patient 7), suffering from West syndrome, who at 3 years of age had an MRI that showed approximately $10 \mathrm{~mm}$

\footnotetext{
* Correspondence: a.panconesi@usl11.toscana.it

Headache Center, Department of Neurology, Health Authority 11, Empoli, Italy
}

Cite this article as: Panconesi et al: P021. Investigation on occipital headache associated with vertigo and vomiting discovers a familial clustering of Chiari I malformation and a "puzzle". The Journal of Headache and Pain 2015 16(Suppl 1):A172. 